\title{
SARC-F test in Sarcopenia and Frailty: A Narrative Review
}

Gülistan Bahat ${ }^{1}$, Duygu Erbaș Sacar ${ }^{1}$

Istanbul University, Istanbul Medical School, Department of Internal Medicine, Division of Geriatrics, Capa, 34093, Istanbul, Turkey

Corresponding Author: Gülistan Bahat, Istanbul University, Istanbul Medical School, Department of Internal Medicine, Division of Geriatrics, Capa, 34093, Istanbul, Turkey

Received date: April 10, 2021; Accepted date: April 15, 2021 ; Published date: April 28,2021

Citation: Bahat G., Duygu E. Sacar (2021) SARC-F test in Sarcopenia and Frailty: A Narrative Review. J.Nutrition and Food Processing, 4(3); DOI:10.31579/2637-8914/047

Copyright: ( ) 2021, Gülistan Bahat. This is an open access article distributed under the Creative Commons Attribution License, which permits unrestricted use, distribution, and reproduction in any medium, provided the original work is properly cited.

\begin{abstract}
SARC-F is a commonly used screening tool for sarcopenia case finding due to its user-friendly and very practical application. It has been introduced to screen for sarcopenia with simple functional questions obviating the need for the measurement of muscle mass. A score equal to or greater than 4 has been reported as predictive of poor outcomes. Sarcopenia is a geriatric syndrome associated with well-known adverse consequences. The growing awareness of sarcopenia as a determinant of poor health in older people has underlined the importance of rapidly diagnosing sarcopenia, which will aid clinicians for implementing prevention and treatment strategies. It has been recommended formal tool for sarcopenia screening/case-finding. In this narrative review, we aimed to evaluate the use of SARC-F, its ability to screen and diagnose sarcopenia and its potential use in the fields other than sarcopenia, i.e. frailty. We conclude that SARC-F stands as one of the most useful and applied tool in studies focusing on screening and diagnosis of sarcopenia. In addition, it has a great potential to be used as a frailty screening tool.
\end{abstract}

Keywords: SARC-F, sarcopenia, frailty, use, screen diagnose; osteoporosis screening score;

\section{Introduction}

SARC-F is a commonly used screening tool for sarcopenia case finding due to its user-friendly and very practical application. It has been first introduced by Malmstrom and Morley in 2013 considering that sarcopenia is definable by simple functional questions, by an analogy to FRAX osteoporosis screening score [1]. Thereby, the authors aimed to obviate the need for the measurement of muscle mass which is the most time-consuming and limiting step while diagnosing sarcopenia. Their preliminary studies given in this first report have suggested that a score equal to or greater than 4 was predictive of poor outcomes $[1,2]$.

Sarcopenia is a syndrome characterized by the loss of skeletal muscle mass, strength, and physical performance. Sarcopenia owns well-known negative consequences i.e., functional dependence, falls/ injuries, institutionalization, and mortality [3]. The growing awareness of sarcopenia as a determinant of poor health in older people has underlined the importance of rapidly diagnosing sarcopenia, because there is increasing evidence that therapeutic interventions can improve outcomes. The clinicians will, thereby, have the chance to introduce prevention and treatment strategies to combat with sarcopenia.

The European Working Group on Sarcopenia in Older People (EWGSOP) diagnostic algorithm of diagnosis of sarcopenia has been widely accepted in the clinical and research community due to its practical recommendations and it is the most frequently used definition for sarcopenia in clinical studies [3]. EWGSOP2 consensus, which is the revision of the first consensus on the definition and diagnosis of sarcopenia published eight years ago, has been recently issued [4]. The
EWGSOP2 definition has provided a better description of sarcopenia and enumerated the methods for use in diagnosing sarcopenia in clinical practice and research activities It presents an updated algorithm for sarcopenia case-finding, diagnosis and severity determination [4]. In the context of EWGSOP2 consensus, the recommended formal tool for screening/case-finding is the SARC-F tool. As such, SARC-F is being more widely used in clinical practice and research studies.

In this article, we aimed to review the clinical use of SARC-F, its ability to screen and diagnose sarcopenia and its potential uses in the fields other than sarcopenia.

\section{SARC-F Tool}

SARC-F has been first presented in the Conference on Sarcopenia Research in 2012 [3] and published later in 2013 by Malmstrom and Morley [4]. It is a 5-item questionnaire - named by an acronym derived from the following five items: Strength, Assistance with walking, Rising from a chair, Climbing stairs and Falls (SARC-F). Each question has three possible answers that grade the difficulty in performing the different tasks (no $=0$, little $=1$ point, or a lot of difficulty $/$ cannot $=2$ points). Falls are scored based on the number of falls within the last year (no fall= zero, 1 3 falls $=1$ point and.$>=4$ falls $=2$ points). The total score ranges from 0 10 , and a cumulative score of 4 is suggested as a screening threshold for sarcopenia in primary care practice [4]. SARC-F is regarded as one of the best tools that can effectively be used to screen sarcopenia in everyday practice [4]. It is originally presented in English. The European Geriatrics Medicine Society, sarcopenia special interest group took the liberty to coordinate translation of SARC-F to multiple European and world 
languages in order to facilitate its use in practice and researches [17]. With this contribution, cultural adaptation and clinical validation of SARC-F has been performed in a variety of languages[5-17]. Among these studies, one study was in the older hip fracture patients [5] and all others were performed in community-dwelling older adults.

\section{SARC-F: sensitivity and specificity to diagnose sarcopenia}

Sensitivity refers to a tools' ability to correctly identify individuals who have the disease, and specificity refers to the ability to correctly identify those who do not have the disease. SARC-F has been reported to have low sensitivity (between 4\%-35\%) but high specificity (between $80 \%$ $99 \%)$ to diagnoses sarcopenia in a variety of studies $(8,10,13,18,19,21)$ when it is used in the way presented in the original study, i.e. with its cutoff $>=4$ points [4]. A recent meta-analysis including 7 studies on 12,800 subjects found the integrated sensitivity of the SARC-F to be $21 \%$ with a specificity of $90 \%$ [19], which further put forwards the relatively low sensitivity of the SARC-F in diagnosing sarcopenia. In a study in Chinese population [8], Woo et al. reported the sensitivity as $3.8-4.8 \%$ and the specificity as $94-99 \%$. Overall, these studies suggest SARC-F having a relatively low sensitivity albeit very high specificity. When probable sarcopenia was considered, we have recently shown that its sensitivity increased with an accompanying high specificity [18].

When a screening test is considered, a general preference is to have a high sensitivity if consequent work-ups after a positive screening is not deemed costly and/or tiresome in terms of physical and psychological demands. As such, a relatively low sensitivity results of SARC-F has been criticized against its use as an ideal sarcopenia screening tool. Observably, the cut-off score that the performer uses to define a positive result will alter the prevalence of positive screening outcomes and thereby, the sensitivity and specificity of a given test. We have recently analyzed the sensitivity of SARC-F by applying different thresholds within this context [20]. A cut-off point $>=1$ increased sensitivity to $84.2 \%$ with accompanying acceptable specificity of $40.5 \%$. We showed that a SARCF cut-off point $>=2$ demonstrated the best synthesis of sensitivity (64.9\%) and specificity $(67.9 \%)$ for detecting probable sarcopenia. Hence, a SARC-F score of $>=1$ has been suggested better for screening and a cutoff point of $>=4$ better for excluding probable sarcopenia [20]. Very recently, Dodds et al. also suggested that a SARC-F cut-off point of one or above improved sensitivity (65\%) with a $72 \%$ specificity [21].

\section{SARC-CalF tool}

As outlined above, SARC-F questionnaire exhibited very good specificity but low sensitivity when used to diagnose sarcopenia. Accordingly, the researcher have attempted to find ways in an aim to increase its sensitivity to be used more confidently in practice and researches.

SARC-F questionnaire addresses muscle function items but, it has no question related to the muscle mass (MM). All consensus reports used in diagnosis of sarcopenia requires identification of low MM to diagnose a confirmed sarcoipenia. Anthropometric measurements are low-costed, practical ways to predict MM. Calf circumference (CC) evaluation is one of the most frequently used anthropometric approach to evaluate MM [3]. As so, Barbosa-Silva et al. proposed a modified version of the SARC-F, SARC-CalF score, to boost performance of original SARC-F in 2016 [23]. It has been proposed as a possible alternative to the SARC-F, with an aim to raise its sensitivity by integrating $\mathrm{CC}$ as a predictor of muscle mass into the muscle functionality domains assessed by the original questionnaire. In their study, SARC-CalF increased the sensitivity significantly, from $33.3 \%$ to $66.7 \%$ while diagnostic accuracy did not decrease considerably (from $84.2 \%$ to $82.9 \%$ ) [23]. After this study, the researches from China and Brazil, also reported that SARC-CalF had better sensitivity to predict sarcopenia when compared to SARC-F, i.e., $20.0 \%$ vs $48.9 \%, 33.3 \%$ vs $66.7 \%$ [23,24]. Another study reported from Slovenia noted the sensitivity of SARC-CalF as $77.4 \%$, which is considerably higher than that of reported for SARC-F in general [25]. Notably, in a community-dwelling Turkish older adults population with a low prevalence of sarcopenia, we found that adding a calf circumference item to the SARC-F tool (i.e. SARC-CalF) increased the specificity and diagnostic accuracy of SARC-F but did not increase the sensitivity [26]. This may be related to the low prevalence of sarcopenia in the study population. Therefore, we suggest that studies that to be performed in community-dwelling older population with lower prevalence of sarcopenia are needed to comment whether use of SARC-CalF would increase the sensitivity of the test when compared to SARC-F in those individuals [26]. We concluide that the ability of the SARC-CalF method to screen for sarcopenia in various populations and settings are required to be tested.

\section{SARC-F+EBM tool (E for elderly and BM for body mass index)}

It has been suggested that the commbination of the SARC-F test with some routine body mass indicating measurements could allow a better case finding. Kurita et al. conceived the SARC-F questionnaire with two routine measures that is associated with sarcopenia, i.e. age $>=75$ and a body mass index $(\mathrm{BMI})<=21 \mathrm{~kg} / \mathrm{m} 2$. This modified version of SARC-F was named as SARC-F+EBM (E for elderly and BM for body mass index) [27]. In their study they included 959 hospitalized Japanese adults with musculoskeletal disease and reported that SARC-F+EBM had a higher sensitivity $(77.8 \%$ vs $41.7 \%$ ) and area under the curve (AUC) value (0.824 vs 0.557$)$ than the SARC-F. Noteworthy, the two questionnaires had similar specificity (69.6\% vs. $68.5 \%$, respectively) [27]. Hence, SARC-F+EBM increased the sensitivity and overall diagnostic accuracy of SARC-F when used to screen for sarcopenia in patients with musculoskeletal diseases. Yet, one should consider that SARC-F+EBM is a new concept and further validation studies in different settings are required to determine the performance of SARC- F+EBM to detect sarcopenia cases.

\section{Use of SARC-F beyond sarcopenia}

Frailty assessment is located in the center of geriatric care to decrease dependency and increased quality of life. It is closely related with sarcopenia, sarcopenia being one of the central components and contributors of this syndrome. The physical phenotype of frailty shows a significant overlap with sarcopenia and sarcopenia is related to the same negative consequences, i.e. falls, fractures, physical disabilities and mortality as does the frailty [4]. A variety of screening tools have been developed and utilized to evaluate frailty evaluation, however none of them is a gold standard. Therefore, in a very recent study we suggested that SARC-F questionnaire may have a poetential to detect frailty. We showed that SARC-F cut-off $\geq 1$ had a sensitivity of $91.4 \%$ and specificity of $44.9 \%$ to screen frailty. SARC-F cut-off $\geq 2$ showed the best balance between sensitivity and specificity with corresponding figures of $74.1 \%$ and $73.7 \%$, respectively. A SARC-F cut-off score $>=4$ performed best to diagnose frailty with considerably high specificity, $92.6 \%$ with a low-tomoderate sensitivity, $46.2 \%$ [22].

\section{Conclusion}

In conclusion, SARC-F is a very practical and user-friendly tool to screen sarcopenia. It has demonstrated its beneficial use in a number of studies proving its ability to predict adverese outcomes related to sarcopenia. Some SARC-F modifications have been proposed to increase its sensitivity as a case finding tool for confirmed sarcopenia that is associated with the presence of low muscle mass. A most recent suggestion is to use alternative SARC-F cut-offs related to the aim of use as screening or diagnosing sarcopenia. SARC-F stands as one of the most useful and applied tool in studies focusing on screening and diagnosis of sarcopenia. 


\section{References}

1. Malmstrom TK, Morley JE.( 2013) SARC-F: a simple questionnaire to rapidly diagnose sarcopenia. J Am Med Dir Assoc; $14: 531-532$

2. Vellas B, Pahor M, Manini T, Rooks D, Guralnik J M, Morley J, et al.( 2013) Designing pharmaceutical trials for sarcopenia in frail older adults: EU/US Task Force recommendations. The Journal of Nutrition, Health and Aging.; 17, 612-618.

3. Cruz-Jentoft AJ, Baeyens JP, Bauer JM, Boirie Y, Cederholm T, Landi F, Martin FC, Michel JP, Rolland Y, Schneider SM, Topinková E, Vandewoude M, Zamboni M(2010); European Working Group on Sarcopenia in Older People. Sarcopenia: European consensus on definition and diagnosis: Report of the European Working Group on Sarcopenia in Older People. Age Ageing. Jul;39(4):412-23.

4. Cruz-Jentoft AJ, Bahat G, Bauer JM, Boirie Y, Bruyère O, Cederholm T, Cooper C, Landi F, Rolland Y, Sayer AA, et al. Sarcopenia(2019): revised European consensus on definition and diagnosis. Age Ageing,. 48(1): p. 16-31.

5. Ha Y-C, Won CW, Kim M, Chun K-J, Yoo J-I.( 2020) SARC$\mathrm{F}$ as a Useful Tool for Screening Sarcopenia in Elderly Patients with Hip Fractures. J Nutr Health Aging;24:78-82

6. Drey M, Ferrari U, Schraml M, Kemmler W, Schoene D, Franke A, Freiberger E, Kob R, Sieber C,(2020). German Version of SARC-F: Translation, Adaption, and Validation. Journal of the American Medical Directors Association S1525861019308710

7. Sánchez-Rodríguez D, Marco E, Dávalos-Yerovi $V$, et al.(2019) Translation and Validation of the Spanish Version of the SARC-F Questionnaire to Assess Sarcopenia in Older People. J Nutr Health Aging;23:518-524

8. Woo J, Leung J, Morley JE. (2014)Validating the SARC-F: A Suitable Community Screening Tool for Sarcopenia? Journal of the American Medical Directors Association;15:630-634

9. Ida S, Murata K, Nakadachi D, Ishihara Y, Imataka K, Uchida A, Monguchi K, Kaneko R, Fujiwara R, Takahashi H.( 2017) Development of a Japanese version of the SARC-F for diabetic patients: an examination of reliability and validity. Aging Clin Exp Res;29:935-942

10. Parra-Rodríguez L, Szlejf C, García-González AI, Malmstrom TK, Cruz-Arenas E, Rosas-Carrasco O.( 2016) Cross-Cultural Adaptation and Validation of the Spanish-Language Version of the SARC-F to Assess Sarcopenia in Mexican CommunityDwelling Older Adults. Journal of the American Medical Directors Association; 17:1142-1146

11. Rolland Y, Dupuy C, Abellan Van Kan G, Cesari M, Vellas B, Faruch M, Dray C, de Souto Barreto P.(2017) Sarcopenia Screened by the SARC-F Questionnaire and Physical Performances of Elderly Women: A Cross-Sectional Study. Journal of the American Medical Directors Association; 18:848-852

12. Yang M, Lu J, Jiang J, Zeng Y, Tang H. (2018.) Comparison of four sarcopenia screening tools in nursing home residents. Aging Clin Exp Res,

13. Kim S, Kim M, Won CW. (2018) Validation of the Korean Version of the SARC-F Questionnaire to Assess Sarcopenia:
Korean Frailty and Aging Cohort Study. Journal of the American Medical Directors Association;19:40-45.e1

14. Kera T, Kawai H, Hirano H, Kojima M, Watanabe $Y$, Motokawa K, Fujiwara Y, Ihara K, Kim H, Obuchi S.(2019) SARC-F: A validation study with community-dwelling older Japanese adults. Geriatr Gerontol Int;19:1172-1178

15. Gasparik A, Demián MB, Pascanu I. ROMANIAN TRANSLATION AND VALIDATION OF THE SARC-F QUESTIONNAIRE. Acta Endocrinol (Buchar). 2020; 16(2):216-222.

16. Krzymińska-Siemaszko R, Deskur-Śmielecka E, KaluźniakSzymanowska A, Styszyński A, Wieczorowska-Tobis K.(2020) Polish version of SARC-F to assess sarcopenia in older adults: An examination of reliability and validity. PLoS One.; 15(12):e0244001. Published 2020 Dec 21.

17. Bahat G, Yilmaz O, Oren MM. et al.(2018) Cross-cultural adaptation and validation of the SARC-F to assess sarcopenia: methodological report from European Union Geriatric Medicine Society Sarcopenia Special Interest Group. Eur Geriatr Med 9, 23-28

18. Bahat G, Yilmaz O, Kilic C et al.(2018) Performance of SARC$\mathrm{F}$ in regard to sarcopenia definitions, muscle mass and functional measures. J Nutr Health Aging.; Epub ahead of print.

19. Ida S, Kaneko R, Murata K (2018) SARC-F for screening of sarcopenia among older adults: a meta-analysis of screening test accuracy. J Am Med Dir Assoc 19(8):685-689.

20. Sacar, D.E., Kilic, C., Karan, M.A. et al. (2021). Ability of SARC-F to Find Probable Sarcopenia Cases in Older Adults. J Nutr Health Aging

21. Dodds RM, Murray JC, Robinson SM, \& Sayer AA. (2020). The identification of probable sarcopenia in early old age based on the SARC-F tool and clinical suspicion: findings from the 1946 British birth cohort. European Geriatric Medicine, 1-9.

22. Bahat G, Ozkok S, Kilic C. et al.(2020) SARC-F Questionnaire Detects Frailty in Older Adults. J Nutr Health Aging

23. Barbosa-Silva TG, Menezes AM, Bielemann RM et al.(2016) Enhancing SARC-F: Improving Sarcopenia Screening in the Clinical Practice. J Am Med Dir Assoc. 2016 Dec 1;17(12):1136-1141.. Epub Sep 17. PMID: 27650212.

24. Yang M, Hu X, Xie L, Zhang L, Zhou J, Lin J, Wang Y, Li Y, Han Z, Zhang D, Zuo Y, Li Y, Wu L.(2018) Screening Sarcopenia in Community-Dwelling Older Adults: SARC-F vs SARC-F Combined With Calf Circumference (SARC-CalF). J Am Med Dir Assoc. Mar;19(3):277.e1-277.e8

25. Urzi F, Šimunič B, Buzan E.(2017) Basis for Sarcopenia Screening With the SARC-CalF in Nursing Homes. J Am Med Dir Assoc; 18:991.e5-991-10.

26. Bahat G, Oren MM, Yilmaz O, Kılıç C, Aydin K, Karan MA.(2018) Comparing SARC-F with SARC-CalF to Screen Sarcopenia in Community Living Older Adults. J Nutr Health Aging. 22(9):1034-1038.

27. Kurita N, Wakita $\mathrm{T}$, Kamitani $\mathrm{T}$, Wada O, Mizuno K. (2019)SARC-F Validation and SARC-F+EBM Derivation in Musculoskeletal Disease: The SPSS-OK Study. J Nutr Health Aging.;23 (8):732-738. 